\title{
Experimental and Numerical Investigations of Slender Body Side Force with Asymmetric Protuberances
}

\author{
K. Kawauchi* ${ }^{*}$ T. Harada ${ }^{\dagger}$, and K. Kitamura ${ }^{\ddagger}$ \\ Yokohama National University, Yokohama, Kanagawa 240-8501, Japan \\ and \\ S. Nonaka ${ }^{\S}$ \\ Japan Aerospace Exploration Agency (JAXA), Sagamihara, Kanagawa 182-8522, Japan
}

Typical space transportation vehicles have various asymmetric protuberant devices on their surfaces, such as cable ducts and side jet motors. Such protuberances, when arranged asymmetrically with respect to the vehicle axis, are known to cause asymmetric vortices that produce side force. In this study, to understand effects of side force and its associated flow fields, both wind tunnel tests and numerical calculations for a slender body with an asymmetric protuberance were conducted at a wind speed of Mach 1.5. The results of computed aerodynamic coefficients are in good agreement with the experimental results and detailed flow structures are provided. In particular, the results revealed that side force was generated by two factors. It linearly increased as vortices detached from the body and nonlinearly increased based on the effects of secondary vortex scale as the angle of attack increased. Additionally, the axial position and azimuthal angle (angle along the circumferential direction around the body axis) of the protuberance strongly influenced side force characteristics. First, the side force was significantly higher when the protuberance was installed in a forward axial position. Second, when the protuberance was installed on the leeward side of the slender body (upper side), the side force increased with the angle of attack. These results are not limited to the presented configuration and can be applied to other rocket designs for cost reduction and safety enhancement.

* Graduate Student, Graduate School of Engineering, 79-5 Tokiwadai, Hodogaya-ku.

$\dagger$ Graduate Student, Graduate School of Engineering, 79-5 Tokiwadai, Hodogaya-ku. AIAA Student Member.

* Associate Professor, Graduate School of Engineering, 79-5 Tokiwadai, Hodogaya-ku. AIAA Senior Member.

$\S$ Associate professor, JAXA, 3-1-1 Yoshinodai, Chuoku, Sagamihara. AIAA Senior Member. 


\section{Nomenclature}

$$
\begin{aligned}
& A o A=\text { angle-of-attack } \\
& C_{A}=\text { axial force coefficient } \\
& C_{P} \quad=\text { pressure coefficient } \\
& C_{Y} \quad=\text { side force coefficient } \\
& e_{\mathrm{T}} \quad=\text { total energy } \\
& F_{X}=\text { axial force } \\
& F_{Y} \quad=\text { side force } \\
& h \quad=\text { height of the protuberance } \\
& L \quad=\quad \text { reference length } \\
& l=\text { length of protuberance } \\
& M \quad=\text { Mach number } \\
& p \quad=\quad \text { static pressure } \\
& P_{\mathrm{r}} \quad=\text { Prandtl number } \\
& P_{0} \quad=\text { total pressure } \\
& q=\text { dynamic pressure } \\
& R e=\text { Reynolds number } \\
& S \quad=\text { reference area } \\
& T=\text { temperature } \\
& U \quad=\text { freestream velocity } \\
& u, v, w=\text { velocity components in cartesian coordinate } \\
& x, y, z=\text { cartesian coordinate } \\
& x_{\mathrm{cg}}=\text { center of gravity coordinates } \\
& V \quad=\text { volume } \\
& \alpha \quad=\text { angle-of-attack } \\
& \kappa=\text { thermal conductivity } \\
& \mu \quad=\text { (molecular) viscosity } \\
& \rho \quad=\text { density }
\end{aligned}
$$


$\varphi \quad=$ azimuthal angle of protuberance or pressure taps

\author{
Subscripts \\ $0=$ stagnation point condition \\ $\infty=$ free stream quantity \\ $c g=$ center of gravity \\ $t=$ turbulence quantity
}




\section{Introduction}

THE space industry is expected to develop safer and cheaper space transportation equipment by accelerating the entry of private entrepreneurial companies. In particular, small rockets are required for accelerating the launch demands of small satellites $[1,2]$. However, as body size decreases due to the restriction of internal area, various protuberances, such as feed pipes, cable ducts, auxiliary devices, and cameras, must be arranged outside of vehicles. Generally, it is rare for slender bodies to generate asymmetric vortices resulting in side forces at low angles of attack $\left(0^{\circ} \leq \alpha \leq 15^{\circ}\right)$ without protuberances [3,4]. However, if a protuberance is attached to a slender body surface, it generates an asymmetric vortex, even at a low angle-of-attack $\left(0^{\circ} \leq \alpha \leq 15^{\circ}\right)$, and various problems are associated with asymmetric vortex influence [5]. For example, more than 30 protuberances would have been installed on the Ares I rocket, of which six types of protuberances would have been arranged asymmetrically, thereby forming an asymmetric flow field and side force [6]. Additionally, asymmetric vortices can engulf the control devices of a vehicle, increasing the risk of the operational control anomalies. Therefore, it is very important to investigate the relationship between protuberances and the resulting side force to guarantee the safety of vehicle launch trajectories. It has also been reported that asymmetric protuberances may be attached to rocket fairings and that the generated side forces can be used to control rockets [7-8]. Side forces have been observed not only as a result of axial asymmetric protuberances on the surface of slender-bodied objects, but of high angles of attack [9-10]. Therefore, in many development projects, aerodynamic loads induced by protuberances have been examined, such as the Epsilon Launch Vehicle in Japan, and Ares I and Ares V rockets in the United States [5-6, 11-12]. However, these studies are considered to be limited in terms of the design of protuberance arrangement. In fact, a basic and systematic aerodynamic study of the acceptable protuberance locations for generic flight vehicles has not yet been conducted. In such circumstances, Harada et al. [13] examined the effects of basic protuberance position on the roll moment for a slender-bodied vehicle using computational fluid dynamics (CFD), although they did not discuss side forces. Additionally, corresponding experiments were not conducted. As mentioned above, the authors of [5-13] only presented aerodynamic characteristics from either wind tunnel experiments or numerical calculations independently, or focused on a specific configuration.

In this study, we constructed a slender body with a protuberance and performed aerodynamic analysis of its side force characteristics, both experimentally and numerically. This study differs from past studies in the following aspects. First, both experimental and corresponding numerical results are analyzed together to provide a detailed description 
of flow mechanisms, vortex structure, and the aerodynamics of a slender body. Second, this study does not focus on specific vehicle geometry, but instead focuses on typical protuberance positions for flight vehicles. We analyzed side force characteristics (a key factor of controllability) at a wind speed of Mach 1.5 for attack angles of $0^{\circ} \leq \alpha \leq 15^{\circ}$. Mach 1.5 was selected because this is the speed at which a rocket is expected to experience its highest dynamic load (i.e., maximum dynamic pressure, $q$ ) [5]. This is considered to be one of the most important Mach numbers during flight for many rockets. Therefore, in this paper, we analyze the basic aerodynamic characteristics of a side force with protuberance layouts installed on a slender body at Mach 1.5 using both experimental and computational approaches. We hope that our work will be applied to various future rocket designs for safety enhancement and cost reduction.

The remainder of this paper is organized as follows. In Section II, we introduce the configurations of the slender body and protuberance considered in this study. Section III discusses wind tunnel and test model information. Section IV describes our numerical methods and conditions. Comparisons and discussion of experimental and computational results are presented in Section V. Finally, Section VI contains our conclusions.

\section{Configurations and Coordinates}

The flight vehicle configuration for this study is a simple slender-bodied rocket with an axisymmetric body (hemispherical cone + cylinder). This basic configuration does not have any protuberances and will be referred to as "No protuberance (No Pro.)," and is symmetric with respect to the body axis (Fig. 1a). The total length of the vehicle from nose to base $(L)$ is $368.0 \mathrm{~mm}$. The diameter of the cylinder $(D)$ is $41.5 \mathrm{~mm}$, fineness ratio $(L / D)$ is approximately 8.9 , and nose half-apex angle is $17.5^{\circ}$. The base area of the cylinder is defined as area $\left(S=\pi D^{2} / 4\right)$. The center of gravity $x_{\mathrm{cg}}$ is approximately $56 \%$ of $L$. The Cartesian coordinate system $(x, y, z)$ is defined such that the origin corresponds to the tip of the body, $x$-axis is oriented along the axial direction of the vehicle, $y$-axis defines the angleof-attack, and $z$-axis is orthogonal to the other two axes.

The protuberance mimics the attitude control device [5] that is typically installed on rockets, as shown in Fig. $1 \mathrm{~b}$. The height of the protuberance is $h / D=15 \%$ (which is equal to its width) [5] and its length $l$ is $0.06 L$. The position of the protuberance is illustrated in Fig. 2a. We changed the position of the protuberance center as follows: i) front, $x / L$ $=0.22$; ii) middle, $x / L=0.55$; and iii) aft, $x / L=0.95$. These three positions were selected because protuberances are typically installed in these positions on rockets. For example, the Epsilon Launch Vehicle has control devices and the Falcon 9 has landing legs near the aft section. Cable ducts, external cameras, and pipe lines are typically located below 
the fairing (front) and around the center of gravity (middle) [5-6, 13-14]. Figure $2 b$ presents the locations of the protuberance with respect to the studied geometry, where $\varphi$ denotes the azimuthal angles as measured clockwise from the $z$-axis in the direction of the $x$-axis. In this study, the azimuthal angles of the protuberance were set to $\varphi=45,90$, and $135^{\circ}$. In other words, the protuberance is always on the positive $y$ side (port side) of the vehicle. As a result, the protuberance is installed asymmetrically with respect to the freestream, as shown in Fig. 2 b.

\section{A. Wind Tunnel Test Description}

\section{Experimental Apparatus}

In this study, experiments were performed at the Sagamihara Campus supersonic wind tunnel of the Institute of Space and Astronautical Science (ISAS) of the Japan Aerospace Exploration Agency (JAXA). This is a blowdownto-atmosphere facility using air from a pressurized plenum as the test gas flowing through a $600 \times 600 \mathrm{~mm}$ square test section. This facility is capable of producing uniform flows at Mach numbers ranging from $M_{\infty}=1.5$ to $M_{\infty}=4.0$, but the data collected during our experiments was acquired at a freestream Mach number of $M_{\infty}=1.5$. The wind tunnel stagnation pressure $p_{0}$ is $196.1 \mathrm{kPa}$ (uniform flow static pressure is $P_{\infty}=53 \mathrm{kPa}$ ), yielding a Reynolds number of 1.38

$\times 10^{7}$, based on the total length $L . M_{\infty}$ and the freestream velocity $U_{\infty}$ were calculated isentropically from the ratio $p_{\infty} / P_{0}$ and stagnation temperature $T_{0}$. In this study, the data at each angle of attack $(\alpha=0,1,2,3,4,5,8,10,12$ and $15^{\circ}$ ) was sampled every $2.5 \mathrm{~s}$ using an apparatus that can change angles of attack automatically. These flow conditions are summarized in Table 1.

The experimental method involved taking the following measurements:

1) Visualization of the flow field using the Schlieren method.

2) Visualization of the surface streamlines using oil flow.

3) Measurement of the forces and moments using a six-component interior balance.

4) Measurement of the pressure distribution on the slender body surface.

1) Using the Schlieren method, the structures of flow fields and deformation of shockwaves can be visualized and analyzed. 
2) To deepen our understanding of the flow field surrounding the slender-bodied vehicle, we visualized the flow using an oil flow, which is a commonly used wall tracing method. The coating solution was prepared by dissolving titanium dioxide in oleic acid and diluting it with liquid paraffin to prepare an oil containing kerosene (titanium dioxide: oleic acid: liquid paraffin: oil $=8: 1: 1: 11)$. This solution was applied to the black model surface. In the oil flow test, the angles of attack were set to $\alpha=0,8$, and $15^{\circ}$ with a run time of $15 \mathrm{~s}$.

3) Measurement of the six-component forces was carried out at a sampling rate of $1 \mathrm{kHz}$ using an interior balance (manufactured by Nissho-Electric-Works Co., Ltd.) attached to the test model. Various force coefficients and moment coefficients were obtained from the balance measurements. In this test, the pressure at the base of the test model was measured using four pressure sensors located $1 \mathrm{~mm}(2.4 \% \mathrm{~L})$ downstream of the base. The average of the four values was calculated. By taking this base pressure into account, the influence of the sting support was accounted for (base pressure correction [14]). Furthermore, this procedure reproduces the actual flight of a slender-bodied vehicle (after engine cutoff).

4) To obtain the pressure distribution along the direction around the slender body axis, eight pressure ports were installed at both $x / L=0.4$ and $x / L=0.8$. Figure 3 presents the configuration of the pressure ports. The circumferential spacing of the pressure ports was arranged with $\varphi=45^{\circ}$ intervals ( $\varphi$ is defined clockwise from the $z$-axis as viewed from the front of the slender body, as shown in Fig. 2b).

\section{B. Test Model}

Figure 4 presents a schematic of the experimental model and protuberance devices (Pro. device) used in our tests. Three depression regions (front, middle and aft) were allocated over the test model surface (Fig. 3) for Pro. device and No Pro. device, which were tested in turn (Fig. 4 illustrates the front protuberance example, where the Pro. device was inserted into the front depression and the No Pro. device was inserted into the other two depressions). By using this configuration, we were able to change the streamwise positions of the protuberance direction. We could also configure its azimuthal angle $\varphi$ by rotating the Pro. device about the $x$-axis.

\section{Numerical Setup}

\section{A. Governing equations}


In this study, the governing equations were the three-dimensional, compressible Navier-Stokes equations:

$$
\begin{gathered}
\frac{\partial Q}{\partial t}+\frac{\partial F e_{k}}{\partial x_{k}}=\frac{1}{\operatorname{Re}} \frac{\partial F v_{k}}{\partial x_{k}} \\
Q=\left(\begin{array}{c}
\rho \\
\rho u_{l} \\
e_{T}
\end{array}\right), F e=\left(\begin{array}{c}
\rho u_{k} \\
\rho u_{l} u_{k}+p \delta_{l k} \\
\rho u_{k} H
\end{array}\right), F v=\left(\begin{array}{c}
0 \\
\tau_{l k} \\
u_{m} \tau_{m k}+\frac{\kappa}{(\gamma-1) \operatorname{Pr}} \frac{\partial T}{\partial x_{k}}
\end{array}\right) \\
\tau_{l k}=\mu\left(\frac{\partial u_{l}}{\partial x_{k}}+\frac{\partial u_{k}}{\partial x_{l}}\right)-\frac{2}{3} \mu \frac{\partial u_{n}}{\partial x_{n}} \delta_{l k}
\end{gathered}
$$

where the subscripts $k, l, m$, and $n$ take on values of $1,2,3$, denoting Cartesian coordinates, $\rho$ is density, $u_{i}$ represents the velocity components $\left(i=1,2\right.$, and 3 correspond to $x$-, $y$-, and $z$-directional velocity, respectively), $e_{T}$ is the total energy, $p$ is pressure, $H$ is the total enthalpy $\left[\rho H=e_{\mathrm{T}}+p\right], T$ is temperature, and all quantities are dimensionless. The working gas was air approximated by the calorically perfect gas model with a specific heat ratio of $\gamma=1.4$. The Prandtl number is $\operatorname{Pr}=0.71$. The molecular viscosity $\mu$ was calculated based on Sutherland's law. The thermal conductivity $\kappa$ was obtained as $\kappa=\mu$. For modeling turbulence, the molecular viscosity $\mu$ was replaced with $\left(\mu+\mu_{\mathrm{t}}\right)$, where $\mu_{\mathrm{t}}$ is the turbulence viscosity given by the Spalart-Allmaras model [15]. Similarly, $\kappa$ was replaced by $\left(\mu+\mu_{\mathrm{t}} \operatorname{Pr} / \operatorname{Pr}_{\mathrm{t}}\right)$, where the turbulent Prandtl number was $\operatorname{Pr}_{t}=0.90$. The Reynolds number was defined as shown in (2).

$$
R e=\frac{\rho_{\infty} U_{\infty} L}{\mu_{\infty}} \frac{a_{\infty}}{U_{\infty}}=\frac{R e_{\infty}}{M_{\infty}}
$$

where $a$ is the speed of sound $(a=\sqrt{\kappa p / \rho}), U_{\infty}$ is the freestream velocity, and $M_{\infty}$ is the freestream Mach number $\left(M_{\infty}=U_{\infty} / a_{\infty}\right)$.

\section{B. Computational methods}

Three-dimensional numerical computations were conducted using the FAST-Aerodynamic Routine (FaSTAR) [16], which was developed at JAXA as a high-speed flow solver for unstructured grids. The system of equations in (1) was discretized using a cell-centered finite volume method. The Spalart-Allmaras one-equation model without a tripping term with rotation correction (SA-noft2-R) $[17,18]$ was used as a turbulence model. The numerical flux for the inviscid term was calculated using a simple low-dissipation advection upstream splitting method type scheme (SLAU) [19] scheme. The Green-Gauss method [20] was used for gradient reconstruction with minmod [21] as a slope limiter. For a time integration method, the lower-upper symmetric Gauss-Seidel scheme [22] was adopted. These calculations all assume a steady flow. 


\section{Computational grids}

HexaGrid [23], an automatic meshing tool developed at JAXA, was used to generate unstructured computational grids based on a hexahedral structure. The computational grid of the No Pro. case is presented in Fig. 5a. The coordinate system was defined as described above. As shown in Fig. 5b, the computational domain is a $20 L \times 20 L$ cube filled with hexahedra, prisms, pyramids, and tetrahedra. The turbulent computations for the slender body were performed using different grids (coarse: 11 million cells, medium: 44 million cells, and fine: 68 million cells). The axial force $C_{A}$ (to be defined later in Section IV-E) was obtained at an angle of attack of $\alpha=15^{\circ}$ with the different grids listed in Table 2, with similar values for the medium and fine grids (error is $0.6 \%$ ), and a different for value for the coarse grid (error is $1.4 \%$ ). This difference was likely caused by insufficient grids surrounding the body, which were unable to properly capture the vortex structures in the wake of the body. Considering its reasonably close agreement with experimental values, the medium grid was used in this study. Furthermore, while the number of cells (medium) is approximately $4.0 \times 10^{7}$ for the No Pro. case, it is $4.4 \times 10^{7}$ for the other three cases (with a protuberance) because of the increased grid density required around the protuberance. To accurately resolve the boundary layer, the first cell height is designed to satisfy the requirement of $y^{+}<1$.

\section{Computational conditions}

The computational conditions were selected to correspond to the wind-tunnel test conditions listed in Table. 1, such as a freestream Mach number of $M_{\infty}=1.5$, Reynolds number $R e$ (based on the length of the body), and angle of attack $\alpha$.

\section{E. Aerodynamic coefficients}

The axial force coefficient and side force coefficient are defined as follows:

$$
\begin{aligned}
C_{A} & =\frac{F_{x}}{q_{\infty} S} \\
C_{Y} & =\frac{F_{y}}{q_{\infty} S} \\
q_{\infty} & =\frac{1}{2} \rho_{\infty} U_{\infty}^{2}
\end{aligned}
$$


where $F_{x}$ and $F_{y}$ are the $x$ and $y$ component forces integrated over the vehicle surface, respectively, $q_{\infty}$ is the freestream dynamic pressure, $S$ is the reference area (based on the base diameter $D$ ), $\rho_{\infty}$ is the freestream density, and $U_{\infty}$ is the freestream velocity.

The pressure coefficient $C_{p}$ is defined as follows:

$$
C_{p}=\frac{p-p_{\infty}}{q_{\infty}}
$$

where $p$ is the local pressure and $p_{\infty}$ is the freestream pressure.

\section{Results and Discussions}

\section{A. Numerical and Wind Tunnel Results}

In this section, the experimental results of the wind tunnel tests for the slender body configuration and corresponding computed results are compared. Figure 6 presents the both the computational and experimental flow fields without any protuberance (No Pro.) and an angle of attack of $0^{\circ}$ at the symmetry plane $(y=0)$. In the top section of the figure, higher density gradient magnitudes indicate shock or expansion waves, and the computed surface pressure distribution (using the pressure coefficient $C_{\mathrm{p}}$, which is defined in (4)) shows that the pressure is high downstream of the shockwave in front of the body, but relatively low downstream of the expansion waves at the fore of the cylinder. In the Schlieren visualizations, compression waves or shockwaves are colored red and expansion waves are colored blue. According to both the computational and experiment results, the positions of the shockwaves in front of the body and expansion waves at the fore of the cylinder correspond to each other. A shock reflection at the wind tunnel wall is also visible, but it only impinges on the sting far downstream of the model. Therefore, its effects can be neglected.

We conducted additional tests with various protuberance configurations and angles of attack. At an angle of attack of $15^{\circ}$, a protuberance of size $h / D=15 \%$ was installed in the front position of the model with $\varphi=90^{\circ}$. The computational results in Fig. 7a reveal the surface pressure coefficient distribution and surface streamlines, as well as the off-surface density gradient magnitude at the symmetry plane $(y=0)$. Figure $7 \mathrm{~b}$ presents the corresponding wind tunnel test results, including both the Schlieren visualization and oil flow visualizations. One can see that the shockwaves on the windward side (lower side in Figs. 7a and 7b) are stronger than those on the leeward side (upper side) at this angle of attack. Shockwaves can also be observed near the protuberance and were successfully captured 
by the numerical calculations. Furthermore, when examining the oil flow test results for the surface in Fig. 7b, separation lines are visible in the vicinity of the center of the model. One can see that the positions of these lines closely match the numerical results (Fig. 7a). A magnified view of the flow field around the protuberance is presented in Fig. 8. A very complex vortex structure, the boundary layer separation caused by the shockwaves, separation line, and reattachment line from the experiments were accurately captured by our CFD model. Specifically, a "horseshoe vortex" [24] was observed in front of the protuberance.

As a quantitative evaluation metric, the axial force $\left(C_{\mathrm{A}}\right.$, which is defined in (1)) was compared between the experimental and numerical results. Figure 9 presents both the results for the relationship between the axial force coefficients and that between the angles of attack. Axial force was selected as a basis of comparison from the six-force and moment components because it is difficult to obtain correct axial forces through numerical calculations. However, the values obtained by our numerical calculations are in good agreement with the results of the wind tunnel tests. All errors are within $3 \%$ and for $\alpha \leq 15^{\circ}$, the error is $0.3 \%$. Based on these results, our flow computations have been validated.

\section{B. Effect of the Angle of attack}

Both the wind tunnel and numerical results for the side force coefficient $C_{\mathrm{Y}}$ on the slender body with the protuberance installed at the front position (front: $x / L=0.15$ ) and without the protuberance (No Pro.) are presented in Fig. 10. Because the maximum error in $C_{\mathrm{Y}}$ between the CFD and wind tunnel (WT) in the No Pro. case was approximately 0.04 at $\alpha=15^{\circ}$, the values obtained by the numerical calculations are considered to be in good agreement with the results of the wind tunnel tests. When the slender body does not have any protuberance (No Pro.), $C_{\mathrm{Y}}$ remains nearly unchanged with respect to the angle of attack because of the axisymmetric configuration.

We will now discuss the front position case (front) in greater detail. The side force coefficient increases nearly linearly with the angle of attack $\alpha$ as long as it is less than $5^{\circ}$. However, as the angle of attack increases further, nonlinearity in the side force can be observed. Figure 11a presents the vorticity distribution when the protuberance is attached to the front position at each angle of attack $\left(\alpha=5,10\right.$, and $\left.15^{\circ}\right)$. The legend in Fig. 11 represents the pressure coefficient $C_{\mathrm{p}}$ and absolute value of the vorticity with ranges of $-0.4-0.0$ and $0.0-0.1$, respectively. At each angle of attack, the vortex separation from the slender body on both the starboard and port sides can be observed. The vortex on the port side is generated by the protuberance and its vorticity is larger than that of the vortex on the starboard side. Focusing on the port side, the vortex generated by the protuberance moves farther from the slender body surface as 
the angle of attack increases. Figure $11 \mathrm{~b}$ presents a vorticity distribution from a perspective view at $x / L=0.8$ on a plane on the forward and starboard side of the slender body. As the angle of attack increases, the vorticities of both the port and starboard sides become larger and the port side vortex moves farther away from the body surface. Because the core of the vortex has low pressure, the port side gradually shows higher pressure. Therefore, side force from the port side to the starboard side is generated and increases linearly.

We will now discuss the starboard side in Fig. 11b. The vortex remains relatively close to the surface of the slender body at any angle of attack. However, a secondary separation and reattachment that forms secondary vortices can be seen for $\alpha=10$ and $15^{\circ}$. Additionally, the size of the secondary vortex is larger at $\alpha=15^{\circ}$ compared to that at $\alpha=10^{\circ}$. In other words, the size of the secondary vortex gradually increases with the angle of attack. Therefore, the side force increases nonlinearly based on the size of the secondary vortices, which reduces the surface pressure on the starboard side further at angles of attack above $5^{\circ}$. This means that the side force linearly increases as vortices detach from the body surface and nonlinearly increase based on the size of the generated secondary vortices.

\section{Effects of the Axial Position of the Protuberance}

Next, we studied the side force at different positions of the protuberance. Figure 12 presents the experimental and numerical side force coefficients for the front, middle, and aft positions with a protuberance of size $h / D=0.15$ at $\varphi=$ $90^{\circ}$. The numerical data agreed well with the wind tunnel data at every position. In the following analysis, $\alpha=15^{\circ}$ is adopted because it resulted in secondary vortices and $C_{\mathrm{Y}}$ nonlinearity. Focusing on the experimental results, a maximum $C_{Y}$ value of 0.44 can be observed at $\alpha=15^{\circ}$ in the front position case, which has a very high value compared to the maximum values of 0.10 (middle) and 0.067 (aft) in the other cases. We now analyze this quantitative increase in $C_{Y}$. In the experimental results, the value of $C_{Y}$ at $\alpha=15^{\circ}$ is approximately 2.6-2.9 times larger than the value at $\alpha$ $=5^{\circ}$ in the aft $\left(C_{Y}=0.026-0.067\right)$ and middle $\left(C_{Y}=0.034-0.10\right)$ cases. However, when the protuberance is installed in the front position $\left(C_{Y}=0.046^{\circ} 0.44\right), C_{Y}$ at $\alpha=15^{\circ}$ is 9.6 times larger than that at $\alpha={ }^{\circ}$. As described above, the value of the side force coefficient varies depending on the position of the protuberance.

Side force does not occur because of a collision of the air flow with the protuberance, but because of the generation of a complicated flow field, such as a large separation region, and the generation of complex vortices. These effects can be observed in visualizations of the numerical and experimental results, such as the oil flow tests and Schlieren visualization results. Fig. 13a presents the vorticity distributions on a two-dimensional plane for each protuberance position at an angle of attack $\alpha=15^{\circ}$. The legend in Fig. 13a indicates the pressure coefficient $C_{\mathrm{p}}$ and absolute value 
of vorticity with ranges of $-0.4-0.0$ and $0.0-0.1$, respectively. We define one type of these vortices as V1, which are a pair of counter-rotating leeside vortices separated from the body, and the other type as V2, which are generated in the wake of the protuberance (only in cases with the protuberance installed). This distinction can be validated from Fig. 13b (the legend shows the absolute value of the vorticity in the range of $0.0-0.1$ ). For the cases without a protuberance installed (No Pro.) and with a protuberance installed in the aft position, symmetrical vortices (V1) that are separated from the body can be observed. For the cases with the protuberance installed in the front and middle positions, the vortices generated by the protuberance (V2) combine with V1. This new combined vortex is defined as V3. As the vortex core of V3 moves away from the body surface, the pressure on the port surface of the slender body under V3 becomes greater than that on the starboard surface of the slender body under V1. Therefore, it is postulated that the side force arises from this pressure differential from the port side to the starboard side.

$\mathrm{V} 1, \mathrm{~V} 2$, and V3, which were observed in the numerical results, could also be confirmed from the experimental results. Figure 14 presents the Schlieren visualization of the wind tunnel test model with the protuberance at the front, middle, aft positions, as well as No Pro. at $\alpha=15^{\circ}$. First, in the cases of (a) No Pro. and the (b) aft position, the separation vortex V1 from the slender body can be observed and the vortex V2 induced by the protuberance cannot be observed. However, in the (c) middle case, the growing and merging vortex V3 can be observed in Fig. 14c, validating the visualization of V3 in Fig. 13. It is believed that this vortex is generated near the shockwave by the protuberance. Because this vortex extends far from the body surface compared to the V1 vortex observed in the No Pro. and aft cases, it can be assumed that this is a merged V3 vortex generated from both V2 and V1 vortices. In the (d) front case, the vortex can be also observed originating from near the shockwave by the front protuberance and vortex V3 is formed above vortex V1 in the same manner as the middle case. However, the asymmetric region of the vortex in this case is larger than in middle case, which is likely caused by the forward vortex interaction created by the protuberance in the front position. In the Schlieren visualization, the flow field was only observed from the sideview direction, but it is important to understand the surface flow field around the slender body, such as the separation line and reattachment line locations on the model from the z-axis looking down (i.e., top view).

An oil flow test was used to investigate the relationship between the detachment of the flow and protuberance position. Figure 15 presents the oil flow test results at $\alpha=15^{\circ}$. These results show the No Pro. case and front position case, which most clearly illustrate the flow asymmetry. In the No Pro. case, both separation lines and reattachment lines can be observed symmetrically across the slender body surface with respect to the center line. A streamline in 
the region between the reattachment lines can also be observed. This streamline exists at a large angle $\left(25-30^{\circ}\right)$ relative to the central axis and appears to be symmetrical between the port and starboard sides. However, in the front case, it can be observed that the port side reattachment line is located farther outside the center of the slender body. In other words, the vortex on the port side moves away from the body surface considerably based on interactions with the separation vortex V1 and the reattachment line is formed closer to the separation line. When considering the port-side streamlines in the region between the reattachment lines, the angle of the streamline $\left(8-13^{\circ}\right)$ compared to the central axis is different from the starboard side $\left(25-30^{\circ}\right)$ because the vortex is detached from the body surface.

Finally, the pressure coefficient predictions surrounding a cross section at $x / L=0.8$ and $\alpha=15^{\circ}$ are presented in Fig. 16. The azimuthal angle $\varphi$ in the figure was defined as the angular absolute distance from the top of the slender body around either the port or starboard sides $\left(\varphi=180^{\circ}\right.$ represents the bottom of the slender body). Recall that the regions between the solid line on the port side and dotted line on the starboard side correspond to the pressure difference between the port and starboard surfaces, resulting in side force. This region becomes larger in the (a) front case compared to the (b) middle and (c) aft cases. Focusing on the starboard side (solid line) in all cases shown in Fig. 16 , the distribution of the pressure coefficients is nearly unchanged based on the position of protuberance, but on the port side (dotted line), the pressure distribution is greatly influenced by the axial position of the protuberance. In particular, the (a) front case indicates that the port side pressure coefficient distribution is much greater when compared to the (c) aft case, meaning the pressure increases as the core of the growing vortex V3 detaches from the body based on the forward installation of the protuberance.

\section{Effects of the Azimuthal Position of the Protuberance}

Wind tunnel tests and computations were performed for the slender body with a protuberance at the front axial position with azimuthal angles of $\varphi=45,90$ (discussed above), and $135^{\circ}$ around the port side of the slender body for each angle of attack. In the cases where the protuberance was installed at the middle and aft of the body, the change in the side force coefficient with the azimuthal angle is not large, so these cases were omitted from further analysis. The side force coefficient results are presented in Fig. 17. The numerical results show relatively good agreement with the experimental results. The side force coefficient $C_{Y}$ is approximately twice as large when the protuberance is installed at $\varphi=45^{\circ}$ (leeward side) compared to when it is installed at $\varphi=90^{\circ}$ (the horizontal position) at any angle of attack. Additionally, $C_{Y}$ is remains nearly constant when the protuberance is installed at $\varphi=135^{\circ}$ (windward side) as 
the angle of attack increases, meaning very little side force is generated. Therefore, by attaching the protuberance to the upstream side, it is possible to dramatically decrease the side force. Conversely, when it is attached to the downstream side, a greater side force is generated. This phenomenon can be explained using the visualization results of the numerical calculations.

Figure 18 presents the vorticity distributions for a series of two-dimensional planes for each protuberance location at an angle of attack $\alpha=15^{\circ}$. The legend in Fig. 18 represents the pressure coefficient $C_{\mathrm{p}}$ and absolute value of vorticity with ranges of $-0.4-0.0$ and $0.0-0.1$, respectively. In the $\varphi=135^{\circ}$ case, we confirmed that the vorticity at the base plane $(x / L=1.0)$ is very similar to that of the No Pro. case at the base plane and that an asymmetrical flow field does not occur in the vorticity distribution, in contrast to the $\varphi=45$ and $90^{\circ}$ cases. Assuming protuberance is located on the upstream side $\left(\varphi=135^{\circ}\right)$, the V2 vortex generated by the protuberance does not interfere with the V1 separation vortex from the slender body. Therefore, the vortex on the port side does not move away from the body to form a V3 vortex. In the $\varphi=45^{\circ}$ case, one can see that the vortex on the port side is larger and moves further away from the body surface compared to the $\varphi=90^{\circ}$ case. Assuming the protuberance is on the downstream side $\left(\varphi=45^{\circ}\right)$, the V2 vortex merges with the V1 vortex closer to the protuberance installed at the front position. Therefore, the V3 vortex grows significantly moves away from body surface, meaning the port side is exposed to comparatively high pressure. Regarding the starboard side at $\varphi=45^{\circ}$, the V1 vortex is forced closer to the body surface and changes its shape based on the V3 vortex on the port side compared to the $\varphi=90$ and $135^{\circ}$ cases. It is postulated that the core of the vortex, which has relatively low pressure, approaches the slender body surface, which lowers the pressure on the starboard side. Therefore, at $\varphi=45^{\circ}$, the side force increases based on the greater pressure difference between the port and starboard sides. To confirm how the pressure distributions of the starboard and port surfaces vary depending on the azimuthal angle of protuberance, it is necessary to analyze these pressure distributions.

Figure 19 presents the pressure coefficient predictions around a cross section $(x / L=0.8)$ with $\alpha=15^{\circ}$ for all azimuthal angles $\varphi$ of the pressure ports. Using $\varphi=90^{\circ}$ as a baseline, there is no significant pressure differential between the port and starboard sides in the $\varphi=135^{\circ}$ case. This indicates that the vortex never detaches and there is little asymmetry between the port and starboard vortices. However, in the $\varphi=45^{\circ}$ case, there is a much greater pressure difference between the port and starboard sides. Specifically, the pressure distribution on the port side has the highest values out of all cases and the pressure distribution on the starboard side has the lowest. It is noteworthy that the variation in the pressure coefficient distribution with respect to the azimuthal angle on the starboard side is greater 
than that on the port side, which has the protuberance. In other words, the pressure decrease caused by the deformed vortex V1 on the starboard side (approaching the surface of the body by vortex V3 on the port side) is more significant than the pressure increase caused by the port-side vortex detaching from the body.

Finally, Fig. 20 presents a 3D iso-surface map of the side force coefficients obtained from the experimental results when the protuberance was installed in the front position. The horizontal axis represents the angle of attack $\alpha$, depth axis represents the azimuthal angle $\varphi$, and vertical axis represents the side force coefficient. A high angle of attack results in increased side force, except at $\varphi=135^{\circ}$. However, as the azimuthal angle decreases, the dependence on the angle of attack gradually increases. Therefore, to reduce the side force in situations where protuberances must be installed on the forward section of slender body vehicles, protuberances should be oriented such that they are exposed to low angles of attack or high azimuthal angles (colored blue in Fig. 20).

\section{Conclusions}

Experimental and numerical studies were conducted on the air flow past a slender body with a protuberance $(h / D$ $=0.15 ; h$ is body height, and $D$ is body base diameter $)$ installed at front $(x / L=0.22, L$ is body length), middle $(x / L=$ $0.55)$, and aft $(x / L=0.95)$ axial positions over the body surface at a wind speed of Mach 1.5. Additionally, the effects of azimuthal angle $\varphi$ (along the circumferential direction around the body axis) were also investigated. First, the numerical results under wind-tunnel conditions showed good agreement with the corresponding experimental results. Based on both sets of results, we investigated the relationship between protuberance position and side force characteristics, and clarified the associated flow mechanisms.

i) The mechanism of side force generation was clearly revealed by the numerical and experimental visualization results. Generally, when a slender body has an angle of attack, a separation vortex V1 is formed by the forward part of the slender body. Additionally, another vortex V2 is generated by a protuberance and merges with vortex V1. As a result, a combined vortex V3 detaches from the slender body surface, particularly on the port side. Because the core of the vortex has relatively low pressure, the pressure on the body surface increases on the port side (where the protuberance is installed). Simultaneously, the vortex V3 at the port side (protuberance side) also affects V1 on the starboard side (no-protuberance side). Then, vortex V1 from the starboard side is deformed by its interaction with V3 from the port side and its core moves closer to the surface of the slender body. This leads to a pressure decrease on the starboard side 
and the pressure difference between the port and starboard sides increases. As a result, a side force from the port side to the starboard side is produced.

ii) The side force is generated by the attached protuberance and increases with the angle of attack. Specifically, it linearly increases for $\alpha \leq 5^{\circ}$ based on vortices detaching from the body surface on the port side. However, it nonlinearly increases for $\alpha>5^{\circ}$ based on the secondary vortex size on the starboard side.

iii) The side force characteristics are greatly influenced by the axial position of the protuberance. For example, when a protuberance is installed at the aft or middle positions, the side force coefficient $C_{\mathrm{Y}}$ grows by approximately 2.6-2.9 times at $\alpha=15^{\circ}$ compared to $\alpha=5^{\circ}$. However, in the front case, $C_{Y}$ at $\alpha=15^{\circ}$ is 9.6 times larger than that at $\alpha=5^{\circ}$ because a hybrid vortex V3 grows and detaches because of the vortex V2 generated at the forward region, which does not occur for the aft and middle cases.

iv) Regarding the front position (where the effect of the axial position of the protuberance is most significant), the effects of the azimuthal angle of the protuberance were investigated. The results revealed that the side force is suppressed at $\varphi=135^{\circ}$ (measured from the leeward side), meaning the side force coefficient is nearly constant at all angles of attack, because V3 is remains relatively close to the body surface. However, at $\varphi=45^{\circ}$, we found that the side force is augmented by the attack angle, showing approximately twice the force compared to the case of $\varphi=90^{\circ}$ at most attack angles. This is because the vortex V3 that is generated by the front protuberance detaches at an early stage at small values of $\varphi$, which affects the leeward flow and raises the pressure there.

We studied a single asymmetric protuberance as a basic research problem. However, real flight designs have multiple protuberance layouts whose effects should be clarified. Therefore, as future work, we will examine multiple protuberances in both the axial and azimuthal directions.

\section{Acknowledgments}

The authors thank the wind tunnel experiment team of Institute of Space and Astronautical Science (ISAS), JAXA, for their experimental setup and cooperation. We also wish to thank the Advanced Technology Group at the ISAS for providing us with an accurate test model and helpful advice. We appreciate Mr. Satoshi Yamauchi of IHI Aerospace Engineering Co., Ltd., Japan, for his cooperation in conducting the wind tunnel tests. For the flow computations presented in this paper, we used the 3D unstructured grid flow solver FaSTAR, which was developed at JAXA. In several numerical tests, the grid generator HexaGrid, which was also developed at JAXA, and JAXA's Super 
Computer 2 (JSS2) were also used. We are grateful for this significant assistance from JAXA. We would like to thank Mr. Richardson Matthew of Tokyo University and Editage (www.editage.jp) for English language editing.

\section{References}

[1] Gamble, K. B., and Lightsey, E. G., "Decision Analysis Tool for Small Satellite Risk Management," Journal of Spacecraft and Rockets, Vol.53, 2016, pp. 420-432. doi: 10.2514/1.A33401

[2] Casalino, L., Pastrone Sutliff, D., Masseni, F., “Optimization of Hybrid Rocket Enginesfor Small Satellite Launchers,” 2018 Joint Propulsion Conference. doi: $10.2514 / 6.2008-4926$

[3] Maynes, R. D., and Gebert, G. A., "Rotating NoseTip Effects on Slender Body Aerodynamics at High Angles of Attack," Journal of Spacecraft and Rockets, Vol. 32, No. 6, 1995, pp. 944-950. doi: $10.2514 / 3.26713$

[4] Kumar, P., and Prasad, J. K., "Mechanism of Side Force Generation and Its Alleviation over a Slender Body," Journal of Spacecraft and Rockets, Vol. 53, No. 1, Jul. 2016, pp. 896-916. doi: $10.2514 / 1 . A 33290$

[5] Kitamura, K., Nonaka, S., Kuzuu, K., Aono, J., Fujimoto, K., and Shima, E., "Numerical and Experimental Investigations of Epsilon Launch Vehicle Aerodynamics at Mach 1.5," Journal of Spacecraft and Rockets, Vol. 50, No. 4, Jul. 2013, pp. 896916. doi: 10.2514/1.A32284

[6] Abdol-Hamid, K. S., Ghaffari, F., and Parlette, E. B., “Ares I Vehicle Computed Turbulent Ascent Aerodynamic Data Development and Analysis,” Journal of Spacecraft and Rockets, Vol. 49, No. 4, 2012, pp. 596-608. doi: 10.2514/1.A32112

[7] Chen, S., Corriveau, D., McIlwain, S., "Numerical Aerodynamic Investigations on Missile Yawing Control using nose-mounted Flow Effevtors," Journal of Spacecraft and Rockets, Vol. 45, No. 2, March-April. 2008. doi: $10.2514 / 1.32549$

[8] Stephen, E. J., Broadbent, D. Q., Matherne, B. A., “Investigation of Reshaping Pin Protuberance for Missile Flight Control in Supersonic Flow" 51st AIAA Aerospace Science Meeting, AIAA Paper 2018-0812, Jan. 2018. doi: $10.2514 / 6.2018-1515$

[9] Corriveau, D., Hamel, N., and Wong, F. C., "Side Force Generation Mechanism for a Missile with Nose-Mounted MicroStructures," AIAA Paper 2006-3000, 2006. 
[10] Applebaum, M., Eppard, M., Hall, L., and Blevins, J., "Protuberance Aerodynamic Loads for Space Launch Vehicle Systems using CFD,” AIAA Paper 2011-3649, 2011.

[11] Hall, R. M. and Bauer, S. X., “Aerodynamic Characterization of the Ares Launch Vehicles,” Journal of Spacecraft and Rockets, Vol. 49, No. 4, July-August 2012, pp. 558-563.

doi: 10.2514/1.A32174

[12] Panda, J., Martin, F. W., and Sutliff, D. L., "Estimation of the Unsteady Aerodynamics Load on Space Shuttle External Tank Protuberances from a Component Wind Tunnel Test," AIAA Paper 2008-0232, 2008.

doi: $10.2514 / 6.2008-232$

[13] Harada, T., Kitamura, K., and Nonaka, S., “Aerodynamic Analysis on Flight Vehicle with Protuberant Devices,” The 31st ISTS Special Issue of Transaction of JSASS (to be published).

[14] Kitamura, K., Fujimoto, K., Nonaka, S., Irikado, T., Fukuzoe, M., and Shima, E., "Wind Tunnel Tests on Aerodynamic Characteristics of Advanced Solid Rocket," JSASS. Aerospace Technology Japan, Vol. 9, 2010, pp. 9-14 (in Japanese). doi: $10.2322 /$ astj.9.9

[15] Spalart, P. and Allmaras, S., “A One-Equation Turbulence Model for Aerodynamic Flows,” AIAA Paper 1992-439, 1992. doi: 10.1016/s1270-9638(97)90051-1

[16] Hashimoto, A., Murakami, K., Aoyama, T., Hishida, M., Ohno, S., Sakashita, M., Lahur, P., and Sato, Y., "Development of Fast Flow Solver FaSTAR,” Journal of the Japan Society for Aeronautical and Space Sciences, Vol. 63, No. 3, 2015, pp. 96105 (in Japanese). doi: $10.2322 /$ jjsass. 63.96

[17] Zhong, L., "Effect of RANS Turbulence Models on Computation of Vortical Flow over Wing-Body Configuration," Transactions of Japan Society for Aerospace Science, Vol.2005, 152-160, 2005. doi: $10.2322 /$ tjsass. 48.152

[18] Spalart, P. and Shur, M., "On the Sensitization of Turbulence Models to Rotation and Curvature," Aerospace Science and Technology, 297-302, 1997. doi: $10.2514 / 6.1992-439$

[19] Shima, E. and Kitamura, K., "Parameter-Free Simple Low-Dissipation AUSM-Family Scheme for All Speeds," AIAA Journal, Vol. 49, No. 8, 2011, pp.1693-1709. doi: $10.2514 / 1 \mathrm{~J} 050905$

[20] Marvriplis, D. J., "Revisiting the Least-Squares Procedure for Gradient Reconstruction on Unstructured,” AIAA Paper 20033986, 2003. doi: $10.2514 / 6.2003-3986$ 
[21] Roe, P. L., "Characteristics-Based Schemes for the Euler Equations,” Annual Review of Fluid Mechanics, Vol. 18, 1986, pp.337-365.

doi: 10.1146/annurev.fl.18.010186.002005

[22] Yoon, S. and Jameson, A., "Lower-Upper Symmetric-Gauss-Seidel Method for the Euler and Navier-Stokes Equations," AIAA Journal, Vol. 26, No. 9, 1988, pp.1025-1026. doi: $10.2514 / 3.10007$

[23] Hashimoto, A., Murakami, K., Aoyama, T., Yamamoto, M., Murayama, M., and Lahur, P. R., "Drag Prediction on NASA CRM Using Automatic Hexahedra Grid Generation Method,” Journal of Aircraft, Vol. 51, No. 4, 2014, pp. $1172-1182$. doi: $10.2514 / 1 . C 032641$

[24] Hunt, J. C. R., Abell, C. J., Peterka, J. A., and Woo, H., "Kinematical Studies of the Flows around Free or Surface-mounted Obstacles; Applying Topology to Flow Visualization,” Journal of Fluid Mechanics, Vol. 86, No. 1, 1978, pp. 179-200. doi: $10.1017 / \mathrm{S} 0022112078001068$ 


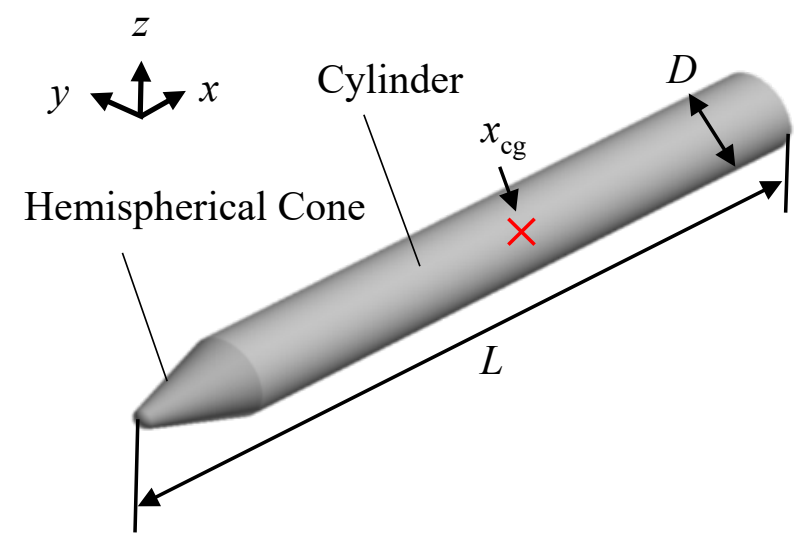

a) Body (Hemispherical Cone + Cylinder) (perspective view).

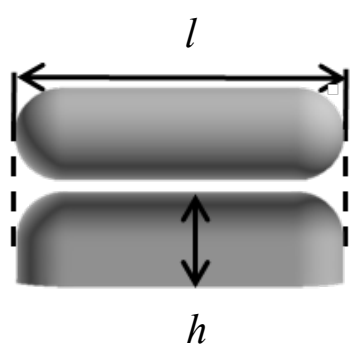

b) Protuberance (side and top view).

Fig. 1 Dimensions of the body and protuberance

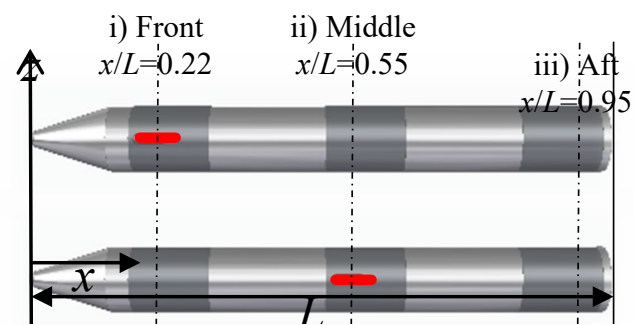

(a) Axial position viewed from the side of the body talled protı

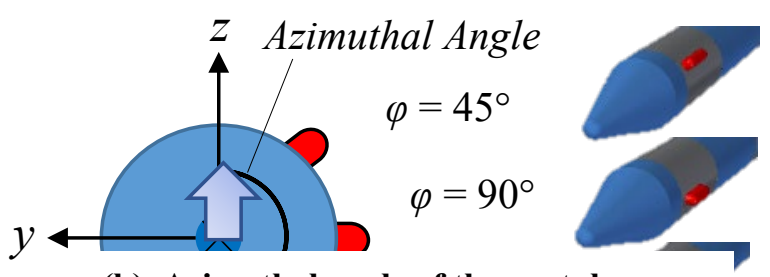

(b) Azimuthal angle of the protuberance viewed from the front of the body

Flow

Table 1 Supersonic wind tunnel test conditions at JAXA

\begin{tabular}{lc}
\hline \hline$M_{\infty}$ & 1.5 \\
$\mathrm{Re}$ & $1.38 \times 10^{7}$ \\
$p_{0}, \mathrm{kPa}$ & 196.1 \\
$\mathrm{~T}_{0}, \mathrm{~K}$ & 294 \\
$\alpha,{ }^{\circ}$ & $0,1,2,3,4,5,8,10,12,15$ \\
Run Time, $\mathrm{s}$ & $15-35$ \\
\hline \hline
\end{tabular}

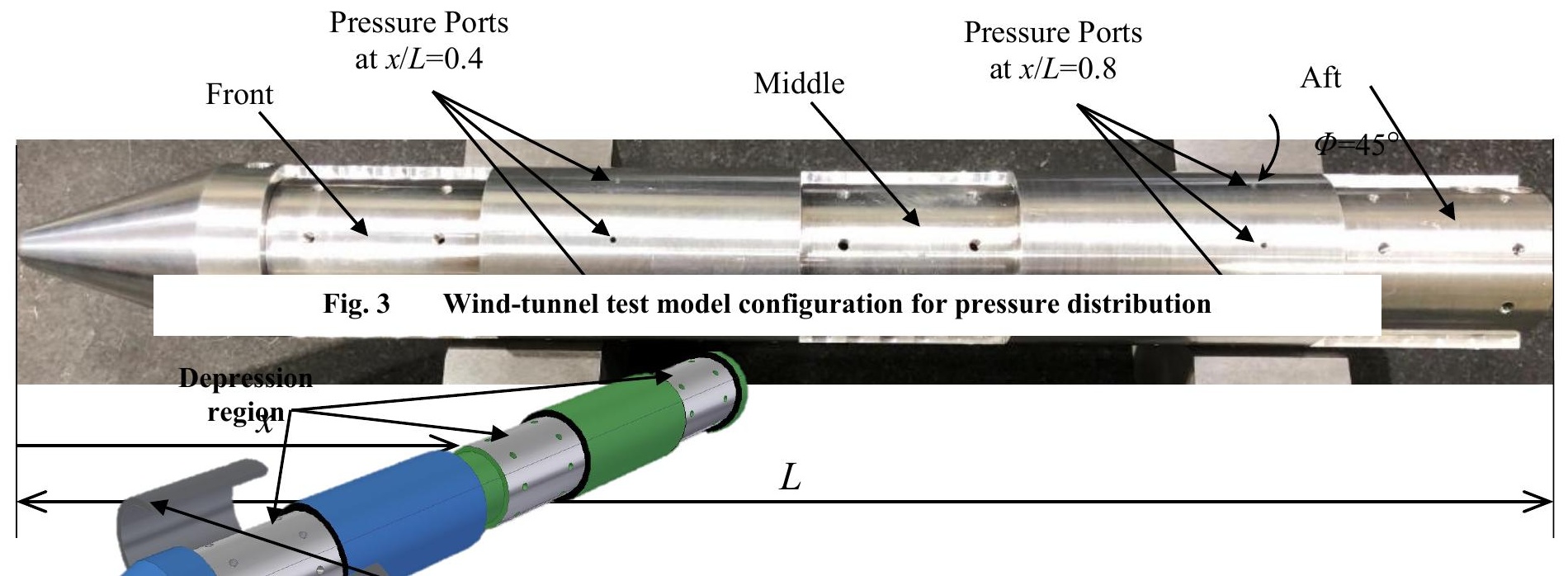


Table 2 Grid sensitivity test for slender body with

\begin{tabular}{ccc}
\hline Grid & Number of cells & $C_{\mathrm{A}}$ \\
\hline Coarse & 11 million & 0.755 \\
Medium & 44 million & 0.766 \\
Fine & 68 million & 0.771 \\
present experiment & $-\cdots-\cdots$ & 0.768 \\
\hline
\end{tabular}

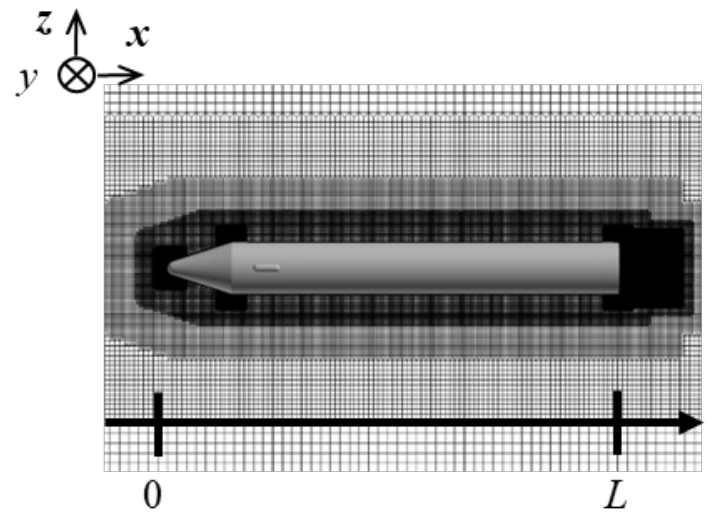

a)

Close-up view: body vicinity.

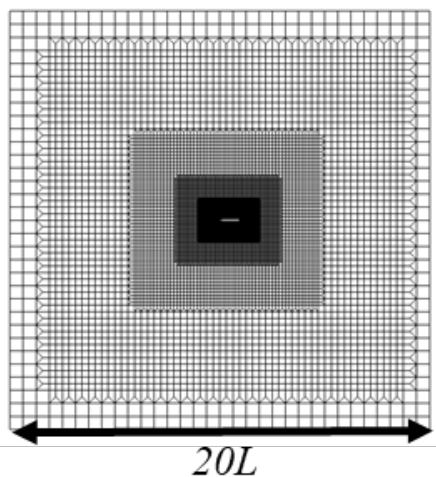

b) Overview.

Fig. 5 Computational 


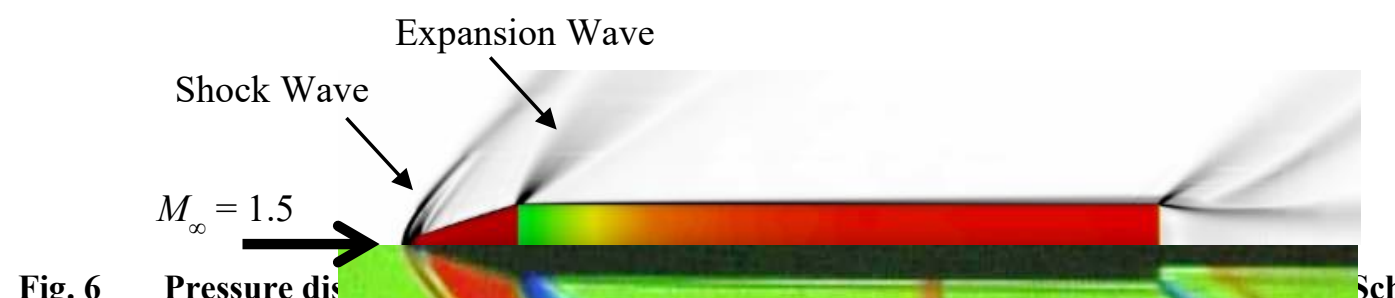

Fig. 6 Pressure dis schlieren
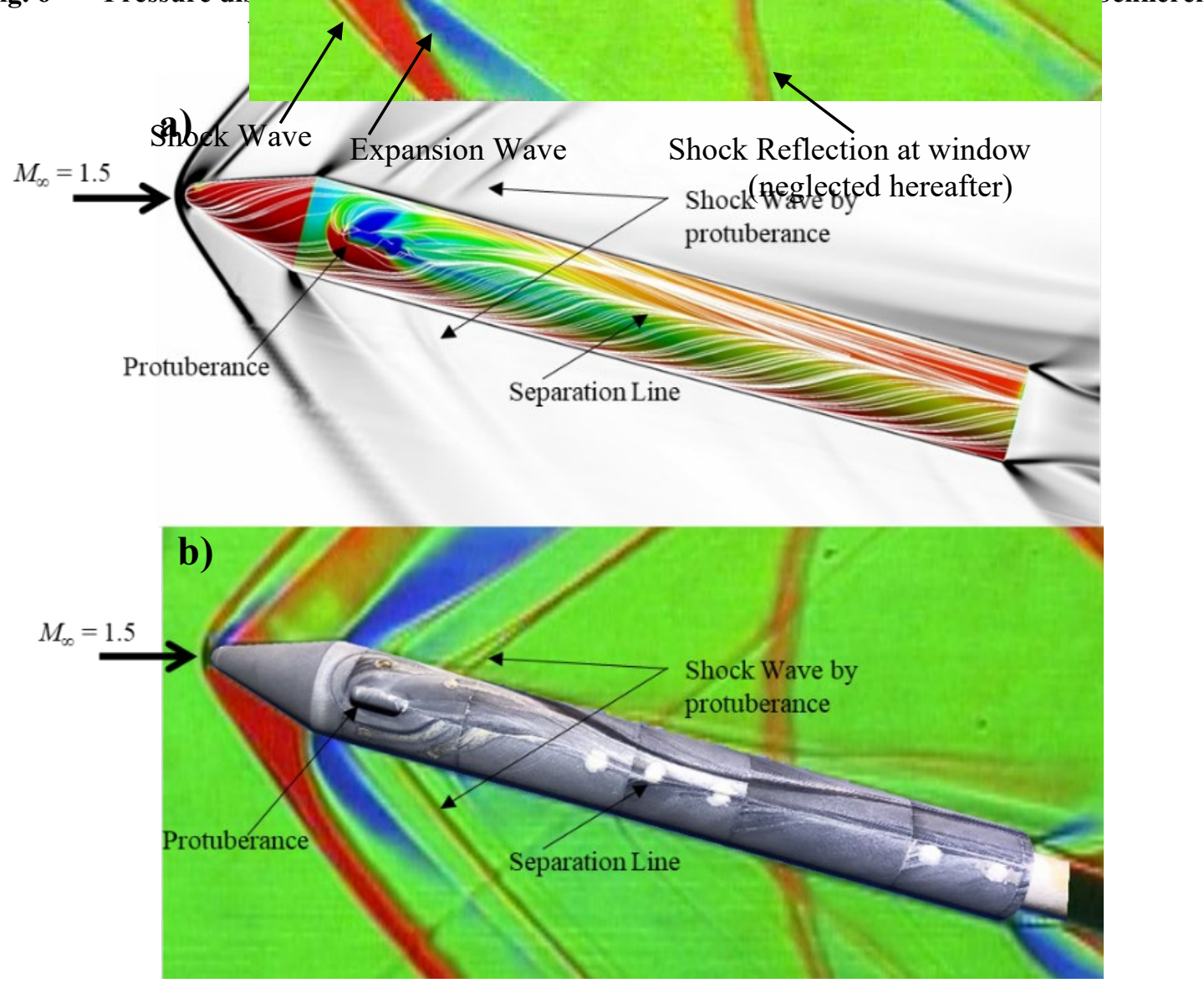

Fig. $7 \quad$ Flowfield of side view (Front case, $\varphi=\mathbf{9 0}^{\circ}, \alpha=\mathbf{1 5}^{\circ}$ )

a) CFD, off-surface: density gradient magnitude; on-surface: computed surface $C_{p}$ and stream-

lines

b) WT, off-surface: Schliren visualization, surface: oil flow image

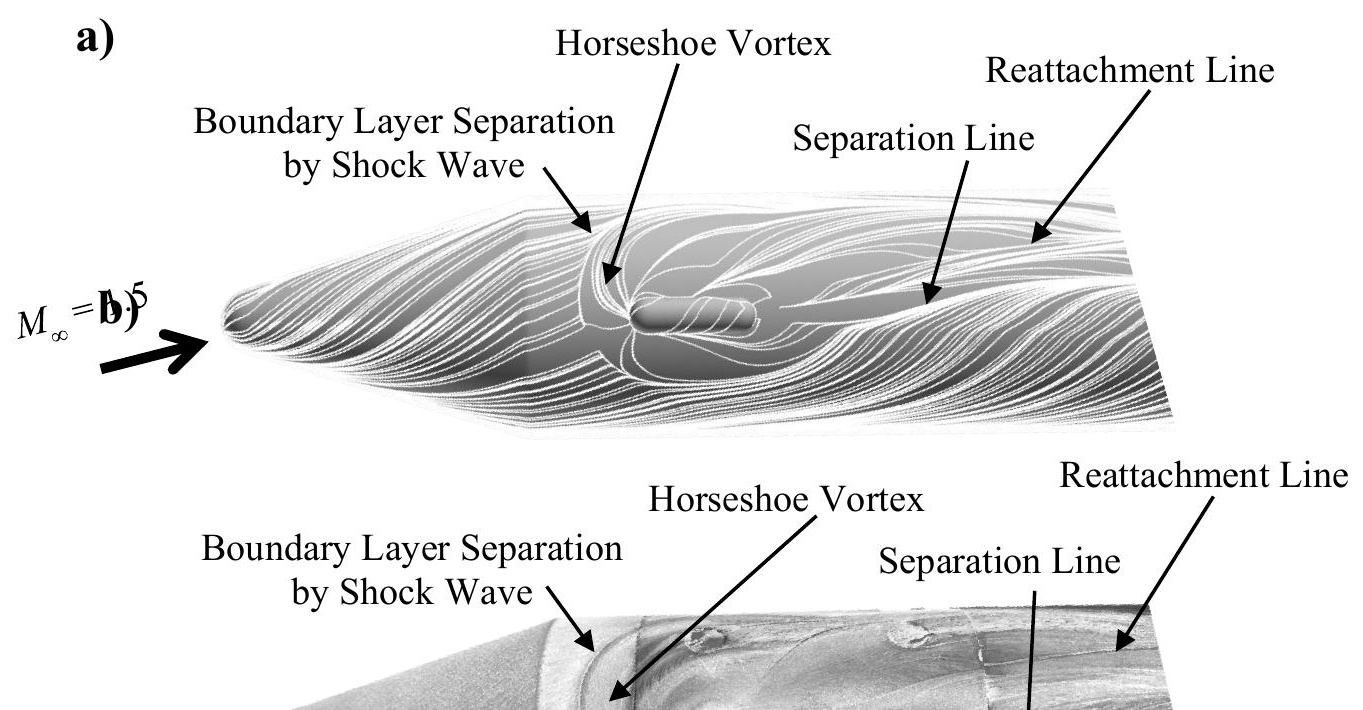


Fig. 8 Side view of streamlines around the protuberance (Front case, $\varphi=90^{\circ}, \alpha=15^{\circ}$ )
a) CFD, streamline
b) WT, oil flow image

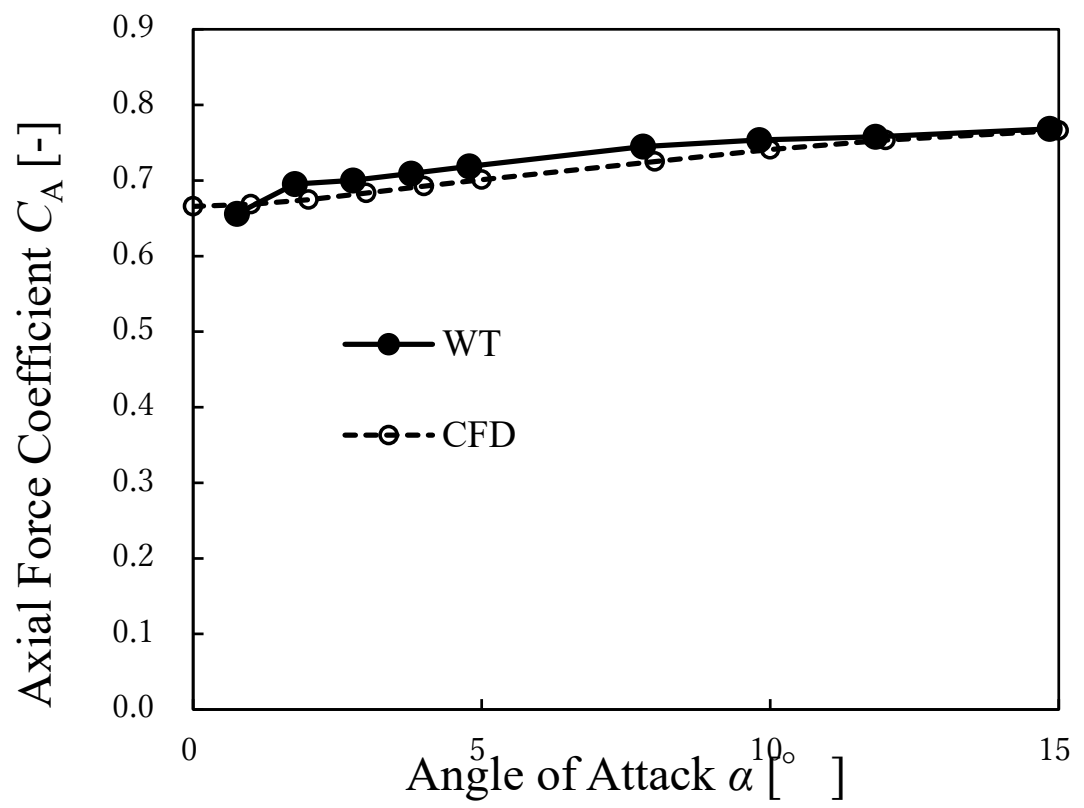

Fig. $9 \alpha$ vs $C_{\mathrm{A}}$ from experimental and computational result.

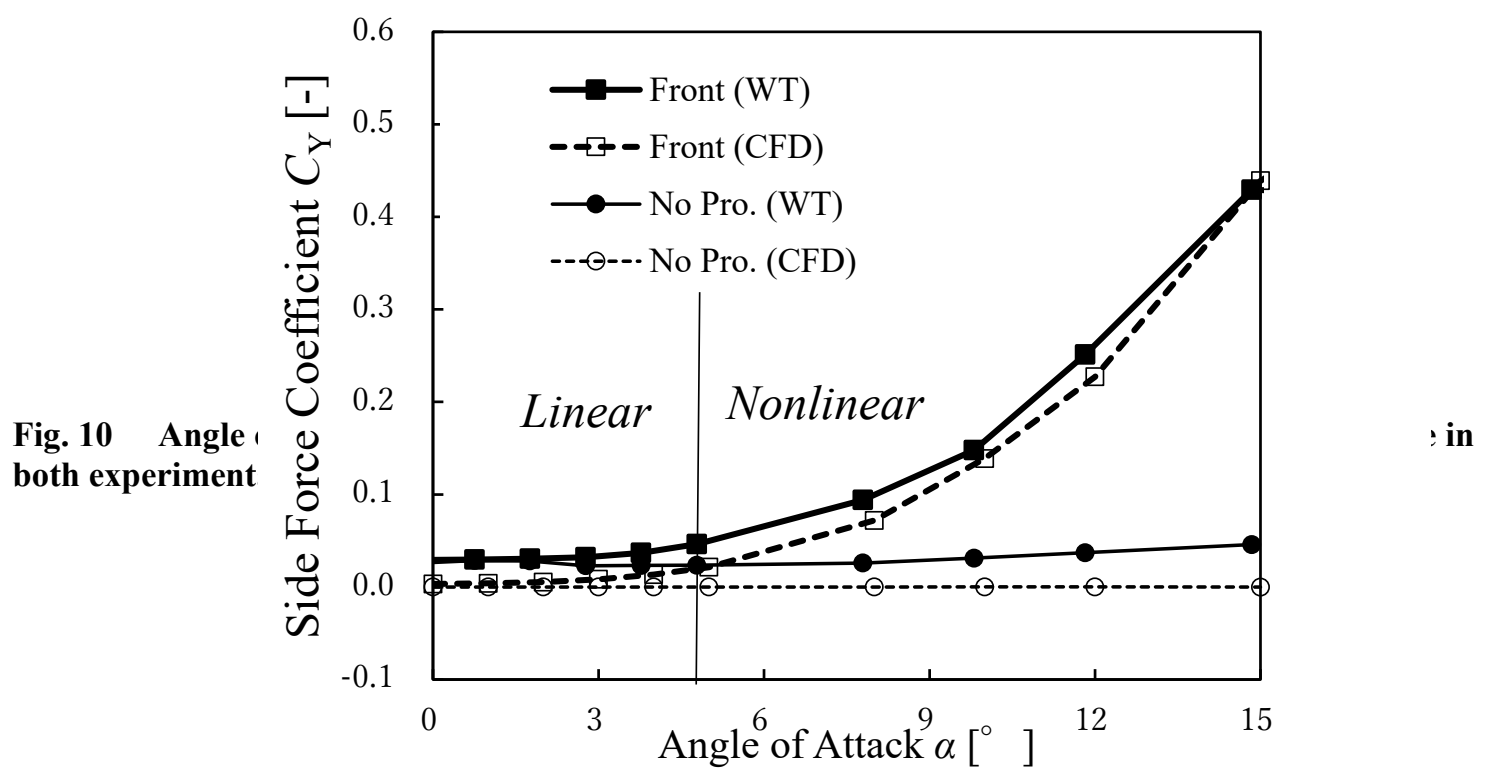



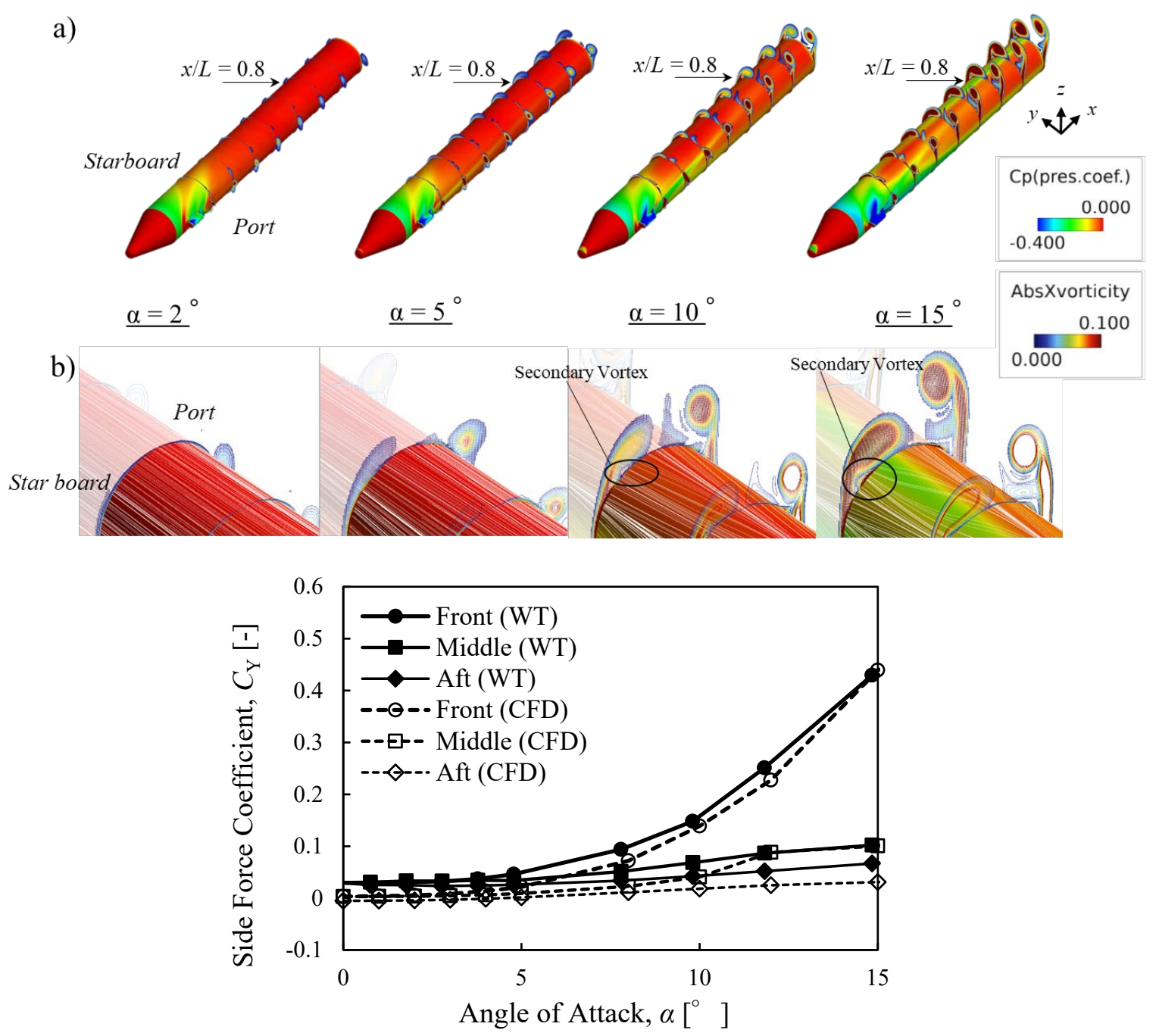

Fig. 12 Angle of attack vs $C_{Y}$ for the axial position of protuberance in both experimental and computational result.

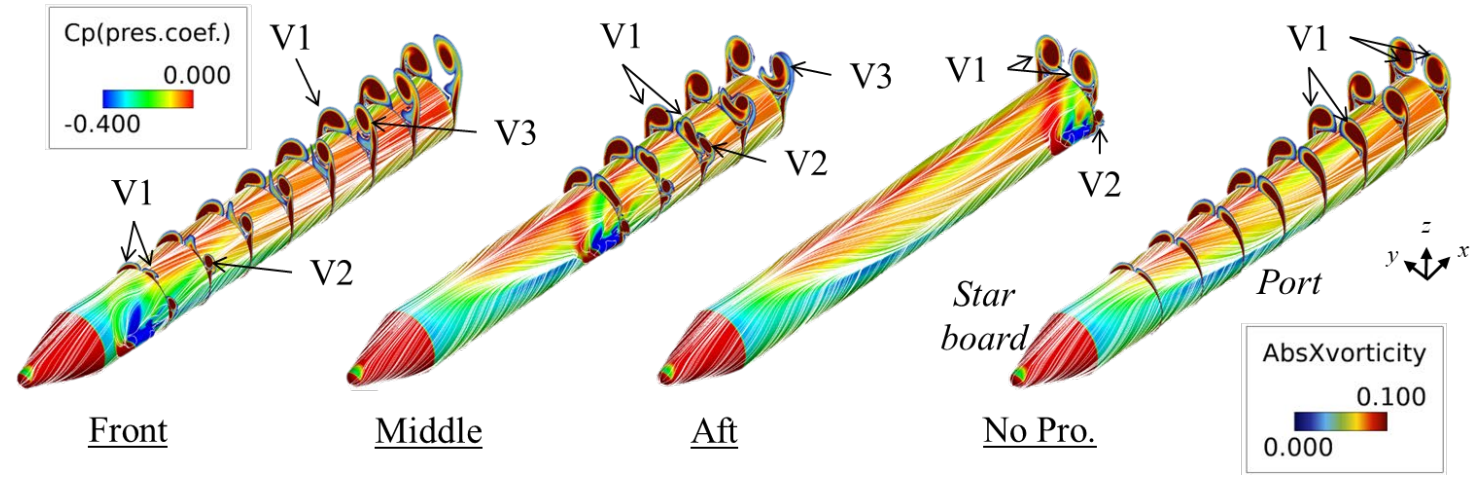

(a) 


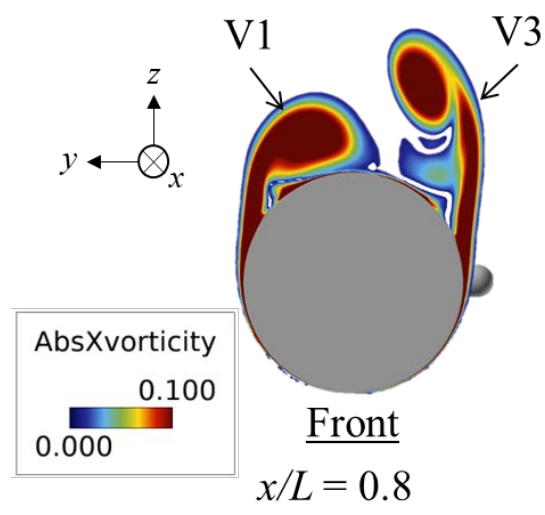

$x / L=0.8$

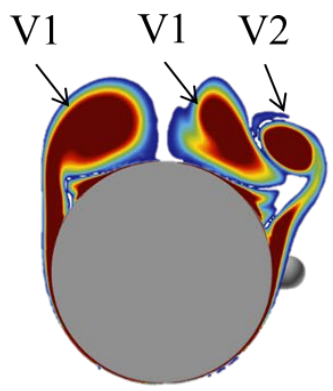

Middle

$x / L=0.8$

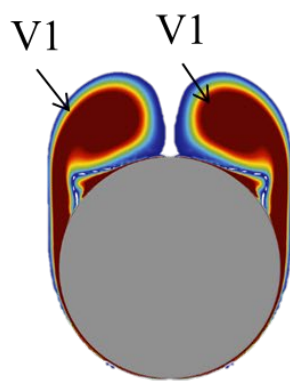

Aft $\quad$ No Pro.

$x / L=0.8$

(b)

Fig. $132 \mathrm{D}(y-z)$ planes colored with vorticity magnitude in each case at $\alpha=15^{\circ}$ : a) planes of $0.1 L$ interval from the protuberance to $1.0 L$ with $C_{p}$ surface contour and the surface flow (from perspective view), b) $x / L=0.8$ plane

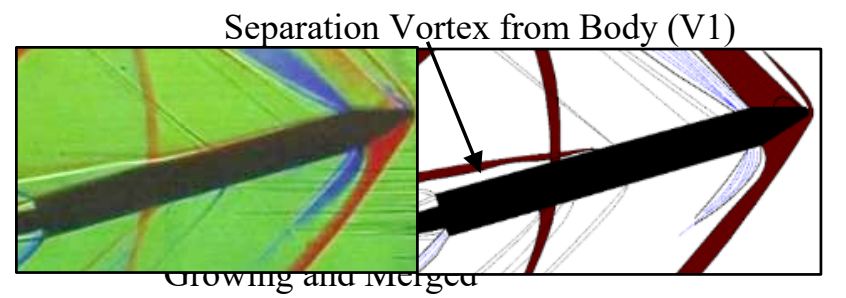

Vortex (V3)

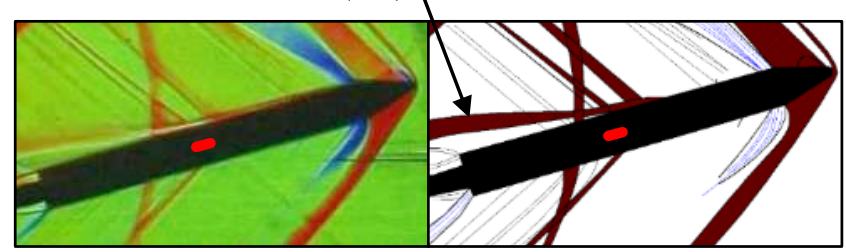

(c) Middle

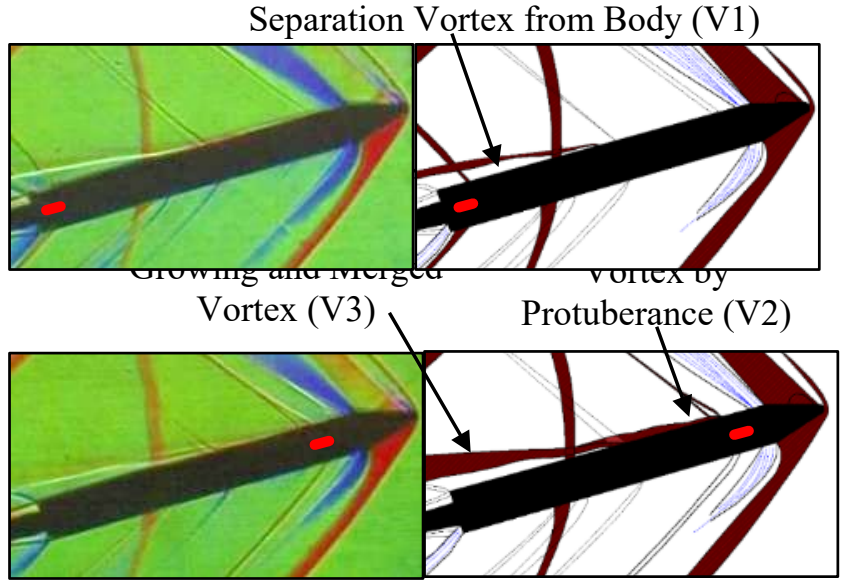

(d) Front

Fig. 14 Schlieren visualization and schematic view $\left(\varphi=90^{\circ}, \alpha=15^{\circ}\right)$ 

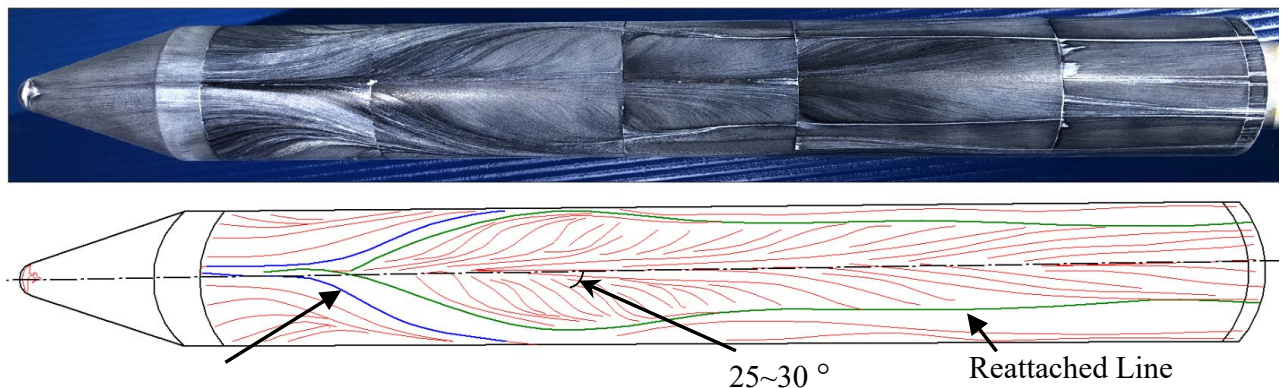

Separation Line

(a) No Pro.
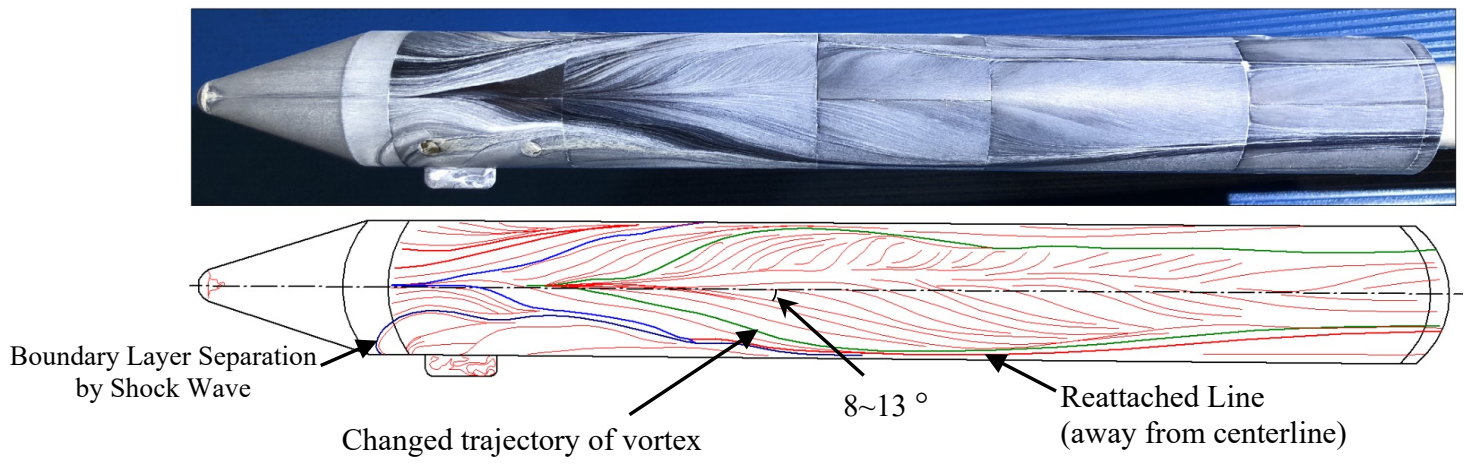

(b) Front

Fig. 15 Oil flow images and schematic view $\left(\varphi=90^{\circ}, \alpha=15^{\circ}\right)$ 


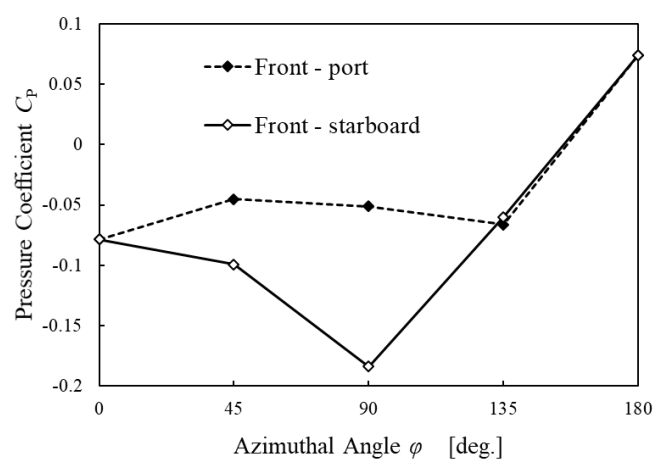

(b) Front

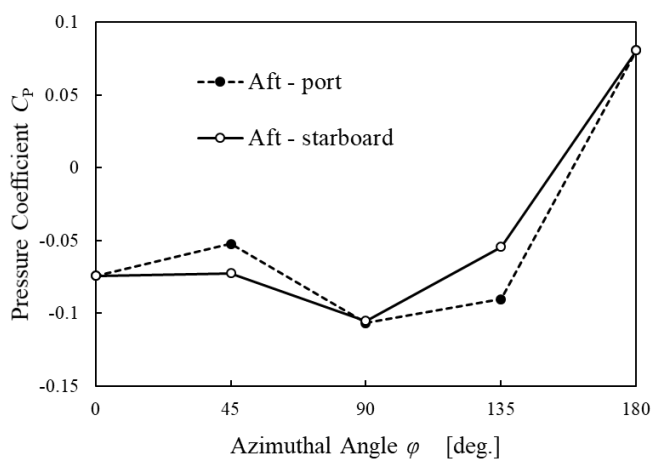

(c) Aft

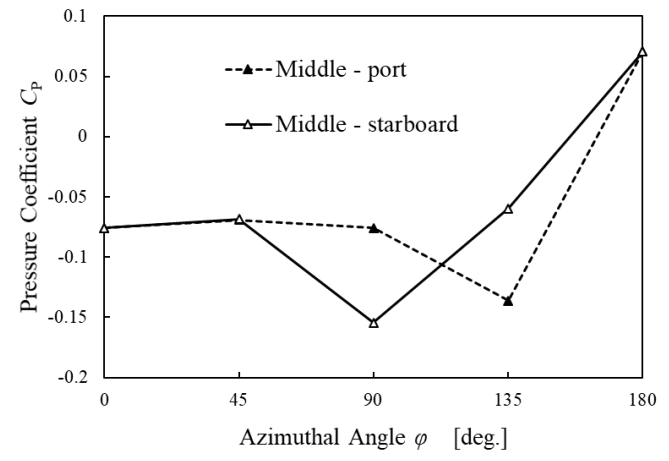

(a) Middle

Fig. 16 Pressure coefficients at $x / L=0.8$ with $\alpha=15^{\circ}$. The solid line corresponding to the starboard side of the body (without a protuberance), and dotted line corresponds to the port side of the body (with a protuberance)

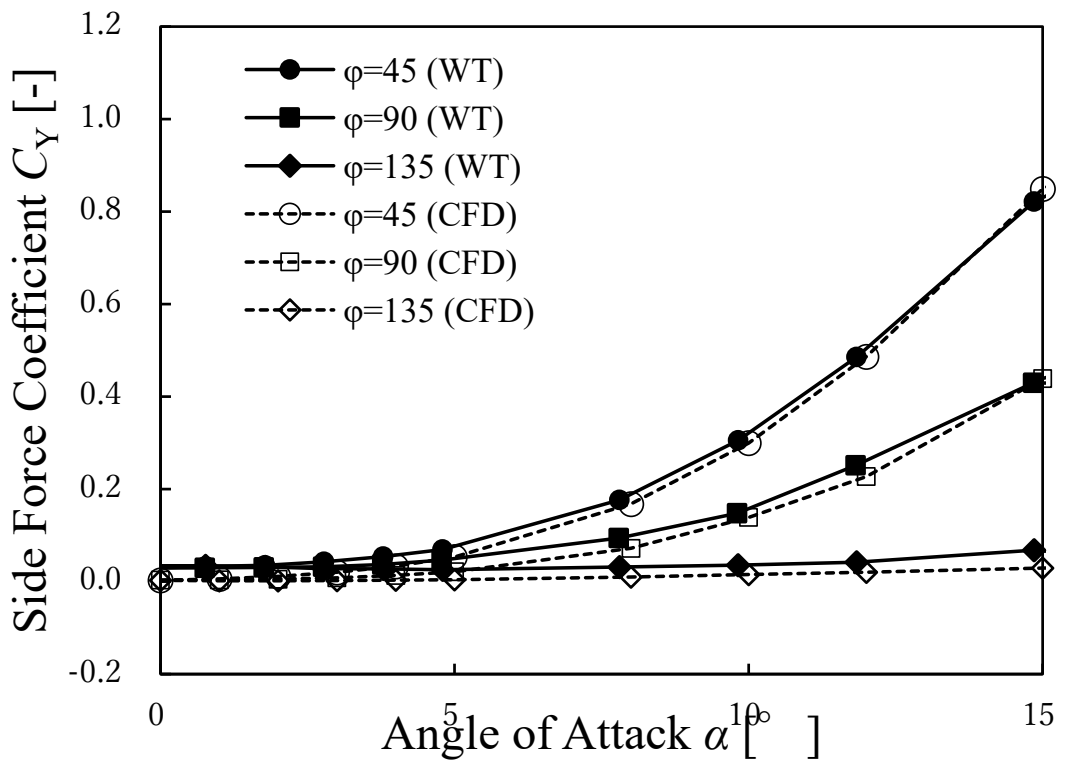

Fig. 17 Side force coefficient vs angle of attack for varying azimuthal angles of the protuberance in both experiment and computation results shown. 


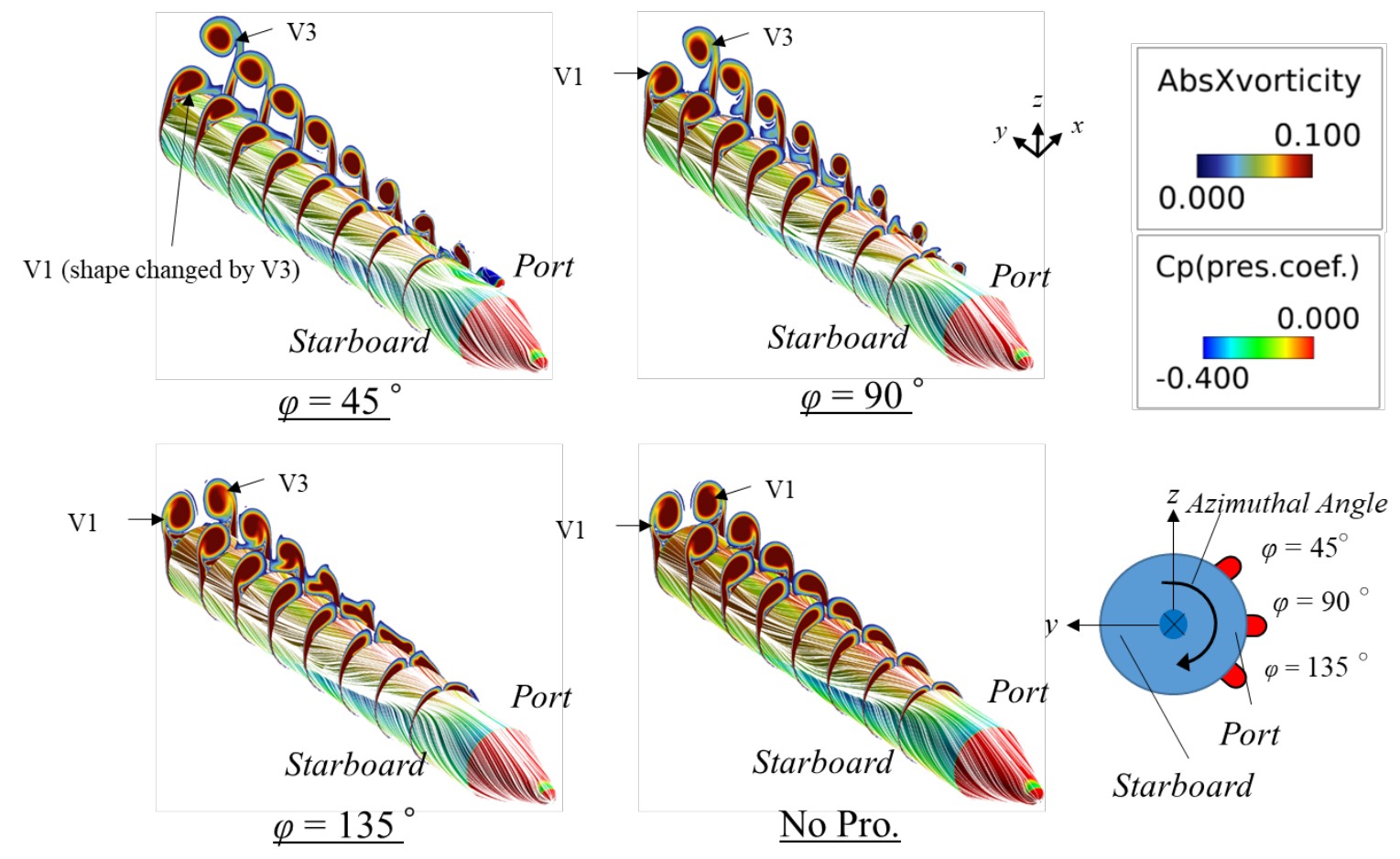

Fig. 18 2D $(y-z)$ planes colored with vorticity magnitude in each case: planes of $0.1 L$ interval from $0.3 L$ to $1.0 L$ with a $C_{p}$ surface contour (perspective view) at $\alpha=15^{\circ}$

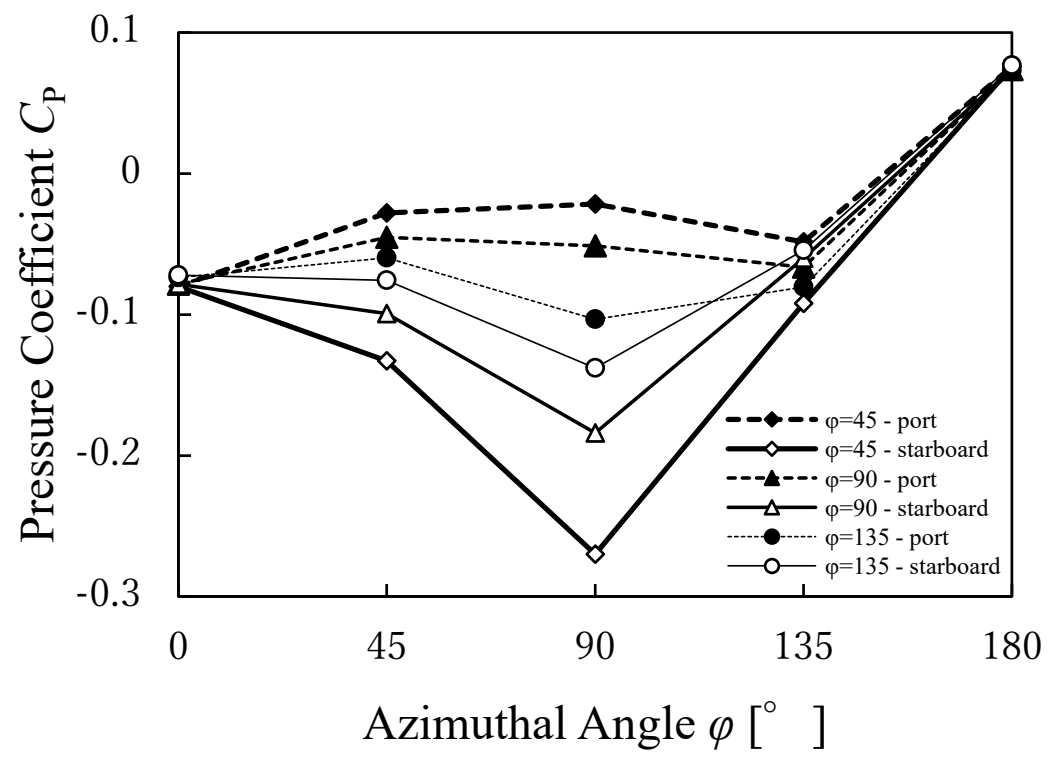

Fig. 19 Pressure coefficients at $x / L=0.8$ with $\alpha=15^{\circ}$. Solid lines correspond to the starboard side of the body (without the protuberance), and dotted line correspond to the port side of the body (with the protuberance installed). 


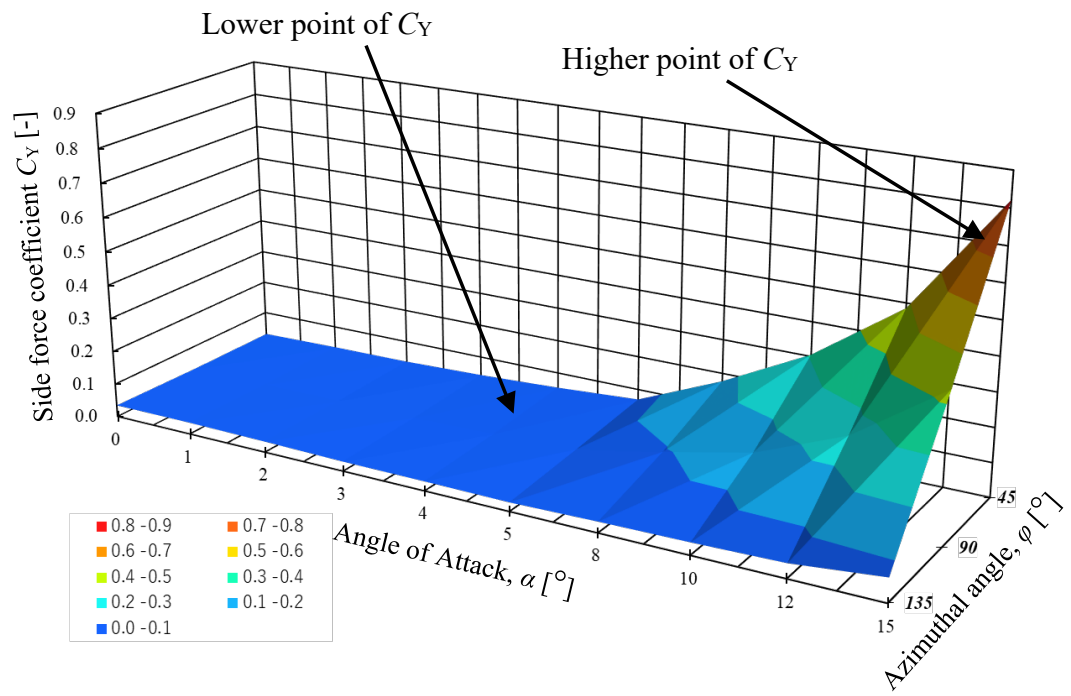

Fig. 20 Side force coefficient vs angle of attack and azimuthal angle of the protuberance 